Tesis. Año 14, 13(16), 2020, 111-126

\title{
La normalidad filosófica, un fenómeno educacional en movimiento
}

\author{
Carlos Miguel Sánchez Paredes \\ cmsanp59@gmail.com
}

\section{Resumen}

En Latinoamérica, la normalidad filosófica objetivada por Romero en 1940 convierte a la filosofía en una función ordinaria de la cultura: la filosofía se convierte en una actividad común y profesional en la cultura. Como consecuencia de ello, asume una función social educacional, comprometida con la educación del pueblo, sin menoscabo de su vocación por el conocimiento y la verdad. Así, se muestra que la normalidad filosófica tiene un doble aspecto educacional: uno "conceptual", que se refiere a la filosofía como actividad intelectual productora de un saber de determinadas características; y, el otro, "administrativo", que implica un ejercicio institucional de la filosofía, vinculado a entidades educacionales o que están involucradas en el sistema formal oficial de educación del pueblo.

Palabras clave: Normalidad filosófica, filosofía latinoamericana, educación.

\section{Abstract}

The philosophical normality objectified by Romero turns philosophy in Latin America into an ordinary function of culture: philosophy becomes a common and professional activity in culture. As a consequence, it assumes an educational social function, committed to the education of the people, without undermining its vocation for knowledge and truth. Philosophical normality has a double educational aspect: a "conceptual", which refers to philosophy as an intellectual activity that produces knowledge of certain characteristics; and the, "administrative", which implies an institutional exercise of philosophy, linked to educational entities or that are involved in the formal formal education system of the people.

Key words: philosophical normality, Latin American philosophy, education. 


\section{La normalidad filosófica, un fenómeno educacional en movimiento ${ }^{1}$}

\section{Introducción}

En diciembre de 1940, tras un prolongado e intenso período de actividad filosófica universitaria y extrauniversitaria que personalizaba ${ }^{2}$ y prenunciaba claramente el pronunciamiento que pronto habría de dar a conocer, el filósofo andaluz-argentino Fran Romero Delgado (1891-1962) manifestó que la evolución histórica de la filosofía en Latinoamérica había llegado ya a una etapa que él denominó de "normalidad filosófica" (Romero, 1961)3. Con esta expresión, Romero quería significar aquella etapa en la evolución de la filosofía en Latinoamérica en la cual la filosofía habíase convertido ya en su percepción, en una función ordinaria de la cultura; es decir, en un componente o actividad habitual de la inteligencia y la cultura propias de cada nación americana.

¿Qué significaba lo anterior? ¿En qué consistía dicha "normalidad filosófica"? ¿En qué sentido postulaba Romero a la filosofía que ya entonces existía en Latinoamérica como una 'función ordinaria de la cultura'? ¿En cuáles indicadores se hubo de basar Romero para acuñar esta categoría de "normalidad filosófica"? ¿Por qué hacia 1940? ¿Tiene esta categoría un rostro educacional? ¿Tiene alguna relevancia esta categoría para el actual pensamiento filosófico en Latinoamérica? Lo que sigue es un ensayo de contestación a cuestiones como las anotadas.

\section{Síntomas de un despertar vocacional}

En efecto, para Romero, hacia 1940, en Latinoamérica acontecía que se había producido un notable incremento del interés por el ejercicio de la actividad filosófica en amplios círculos sociales no necesariamente restringidos al ámbito eclesiástico, sino, más bien, ampliados a la órbita del mundo civil ${ }^{4}$. Así, venía siendo vasto el número de personas que, poco a poco, conforme la filosofía podía ser entronizada y difundida en la escena sociocultural latinoamericana, mostraban adhesión a ella e interés en su cultivo. Es decir, se asombraba 
Romero de que el interés por la filosofía en Latinoamérica sea ya tan grande que parecía haberse convertido en un componente más del complejo panorama cultural latinoamericano. $\mathrm{O}$, como él mismo señala: en una función ordinaria de la cultura.

¿Sobre la base de cuáles indicadores postulaba Romero que Latinoamérica había alcanzado ya aquel estado de "normalidad filosófica"? Como principales indicadores de dicho incremento del interés por la filosofía en Latinoamérica, Romero (1961, pp.150-1) enumeraba los siguientes: (1) la frecuente aparición de artículos filosóficos en revistas especializadas, así como de libros consagrados a dicha temática; (2) el incremento del número de lectores interesados en los escritos de corte filosófico; (3) la posibilidad de que cualquier persona pueda recibir formación filosófica en instituciones educacionales no necesariamente regentadas por la Iglesia; (4) la expansión de las relaciones y vinculaciones académicas entre los interesados en las materias filosóficas ${ }^{5}$, fomentándose así, las redes intelectuales de que habla Randall Collins (2005).

Según la conceptualización romeriana, todos estos indicadores configuraban el atributo más importante del estado de normalidad filosófica, el cual venía dado por el hecho de que la filosofía se hubo de convertir en una "función ordinaria de la cultura”. ¿Qué significa esto?

\section{La filosofía como función ordinaria de la cultura}

A juzgar por la definición romeriana de normalidad filosófica (1961, p. 151), el que la filosofía en Latinoamérica alcance su estado de normalidad, significa que se había convertido en una "función ordinaria de la cultura". Antes, sobre todo hasta el siglo XIX, dice Romero, la filosofía venía siendo un ejercicio en solitario, poco relacionado con otros sujetos filosofantes, entre pares (prenormalizado). Pero, el progresivo y notable incremento en el número de sujetos interesados en la problemática filosófica fue creando lo que Romero denomina el "clima filosófico" que caracterizará al estado de normalidad alcanzado por la filosofía en Latinoamérica.

Dicho "clima filosófico" se configura como una suerte de "opinión pública" especializada en los asuntos filosóficos, y denota: debate público, colectivo filosófico, la formación de corrientes de opinión filosófica, ágora, libre examen y libertad de pensamiento. Así las cosas, quienquiera puede participar de aquello y contribuir a la creación — por tanto- de una cultura filosófica y, en consecuencia, sentar los fundamentos de una tradición de pensamiento de jaez abierto, permanentemente enriquecida.

Fueron diversos los factores que han debido de jalonar la estructuración de ese estado de normalidad filosófica. Durante el siglo XIX, y lo que llevó transcurrido del siglo XX, se produjo una gran diversidad de hechos-factores: 
la influencia intelectual del positivismo y del espiritualismo, el creciente acceso a los libros a una mayor literatura especializada - especialmente científica y filosófica- y la cultura proveniente del Viejo Mundo, con la consiguiente mayor occidentalización de la esfera cultural latinoamericana, las visitas emprendidas por Ortega y Gasset a Latinoamérica, que dieron un espaldarazo en los combates ideológicos contra el positivismo, la reforma universitaria de 1919, que avivó notables cambios en todo el escenario de la educación latinoamericana, la redefinición de los proyectos educacionales nacionales, que generó una mayor apertura a la influencia de la filosofía en la formación de la espiritualidad latinoamericana. Estos, entre otros, han constituido influjos directos.

En el contexto de la postulación romeriana, que la filosofía se convierta en una "función ordinaria de la cultura" significa que la filosofía adquiere una carta de ciudadanía, de inserción institucional con personería propia en el complejo sociocultural. Encuentra en el andamiaje cultural vigente un espacio al cual se adscribe y en el cual y desde el cual puede ser profesionalmente ejercida. La particularidad de ese giro institucional experimentado por la filosofía en Latinoamérica en el siglo XX estribará en que se adjudica de un status de profesión que, entre otras cosas, demandará dedicación exclusiva, un trabajo intelectual de un carácter más técnico, e implicará, inclusive, la asunción de la condición de asalariado por parte del filósofo profesional.

En el complejo andamiaje ideológico de la cultura, entonces la filosofía pasará a ocupar un espacio propio, teniendo la particularidad de haberse convertido en un oficio que habrá de demandar preparación y dedicación; todos los ingredientes de un saber que se ha adjudicado ya del status de profesión, convirtiéndose en una moderna especialidad (Cf. Becher, 2001), a despecho del carácter esencialmente totalizador inherente al saber filosófico.

La presencia institucional de la filosofía, como formando parte del complejo sociocultural latinoamericano, demarcará, asimismo, la naturaleza e intensidad del "clima filosófico". Este "clima filosófico", no obstante, no es estático y no siempre tiene por qué ser el mismo, puesto que no es unívoco en toda época, como tampoco es invariable el grado de acogida o rechazo a dicha presencia sociocultural de la filosofía. La del filósofo es, como ya lo ha mostrado Canfora (2002), una ocupación peligrosa. Si es crítica genuina, como que en verdad la filosofía lo es, entonces siempre será un saber refractario a la sociedad y la misma cultura. En realidad, a esto es que se refiere el profesor español García Norro (2012, p. 31) cuando señala que, por su propia naturaleza, la filosofía no se puede institucionalizar en una sociedad que la estigmatiza o rechaza.

Por ejemplo, no es que hacia 1940 — fecha en que Romero realizó su diagnóstico- la normalidad filosófica era ya un hecho consumado ultimistamente con la filosofía plena e irrecusablemente insertada a la sociedad nacional latinoamericana como una función ordinaria de la cultura, en una doble dimensión 
educacional, como habremos de ver luego ${ }^{6}$. Se había institucionalizado, y su profesionalización fue gradual, sí; y este hecho empezaba a garantizarle cierta estabilidad vía la enseñanza. Abriendo la posibilidad de que, al ser enseñada oficialmente en instituciones educacionales — sobre todo de inspiración más civil-, los filósofos puedan encontrar una fuente de empleo y vivir de aquello para lo que técnicamente se habían formado. Por tanto se fue imponiendo un régimen disciplinar de cuño laical.

La ordinarización de la filosofía podría incluso ser reveladora de que alguna aceptación sociocultural podía estar ganando la filosofía en el conjunto social latinoamericano. Sin embargo, para que la filosofía llegue a convertirse plenamente en una "función ordinaria de la cultura", no ha de bastar con su institucionalización: será menester que ella, la filosofía, sea mínimamente aceptada; es decir, que el conjunto social la acepte como una necesidad sociocultural. Aunque esto suene irrealizable, como utopía que es, moverá la marcha del pensamiento latinoamericano. Aunque acaso, como decía Žižek (Badiou y Žižek 2011, p. 62), la filosofía sea la anormalidad por antonomasia.

Empero, sin duda, al insertarse la filosofía en la cultura como una de las funciones habituales de esta, contribuye a hacerla más compleja e intelectualmente más rica en posibilidades, y también a desarrollarla, manteniéndola vigente. En la medida que el filósofo asume que su atributo sociocultural es ser un pensador de oficio, como diría un poco irónicamente Kant (Kritik, B871), puede, por consiguiente, convertir también a la propia cultura y al conjunto social, en objetos teóricos de dicho "pensar filosófico". Cuando esto ocurre, la filosofía contribuye a la complejización de la cultura, porque, pensándolos, moviliza la libertad humana y todas las competencias y capacidades en favor del desarrollo cultural. De ahí que la filosofía se incorpora a la cultura orgánica como lo que es, un saber esencialmente teórico; aunque sin alejarse de la cotidianeidad, o sin abdicar de las consideraciones morales o políticas que siempre implican a los filosofemas, por muy abstractos y abstrusos que se les presuma ser. Como anotaba el propio Romero en 1943, “el que la filosofía sea teoría estricta, no supone su alejamiento de la vida; antes se integra fecundamente en ella solo en cuanto es teoría, en cuanto obedece a su índole propia (1957, p. 119).

Lo anterior configurará, en rigor, un vínculo fuerte con la sociedad; de modo que el filósofo no sea concebido - en el contexto de la cultura a la cual oficialmente se ha incorporado- como un soñador que refugia su desencanto de lo inmediato en un paisaje de fantasía o en ficciones antes que en los contenidos concretos de la cultura de la que ya forma parte ${ }^{7}$. Así, la presencia institucional de la filosofía en la cultura puede garantizar reproducibilidad teórica creando más teoría y más cultura, así como mecanismos de crítica que pauten una creación cultural más ligada a las tradiciones propias de la cultura. El resultado de esta presencia de la filosofía en la cultura es la mayor humanización 
del ser humano. Como señala Romero, mediante dicha creación, el ser humano "va realizando su ser propio y hace posible que sus descendientes sean cada vez más hombres" (Op.Cit. p. 121).

La filosofía, en tanto que común función cultural, puede tornarse en conciencia de esta, pensándola y repensándola, esparciendo de filosoficidad su contenido. La asunción de su espacio sociocultural propio es una forma de empoderamiento, en el cual y desde el cual ella es lo que es según su ipseidad de saber teórico. Porque, si la cultura consta de muchos aspectos: el saber, las costumbres, las creencias, la moral, la religión, el lenguaje, el derecho, las artes, la ciencia, las instituciones, las técnicas, etc., entonces la filosofía asumirá, frente a todo ese complejo cultural, su naturaleza crítica y problemática, siendo también parte autocrítica de la cultura. Si esto faltase, entonces, como indica Romero, el ser humano:

viviría ingenuamente la cultura, como el animal vive ingenuamente su mundo o ambiente natural. Pero el hombre no se contenta con nada dado, aunque sea él mismo quien lo produce. Quiere que todo ante él se justifique, hasta lo que es producto de su propio espíritu. (Íd., pp. 121-2)

Como hemos dicho, en tanto que fenómeno sociocultural, la normalidad filosófica abre la posibilidad de que se perfile un status ocupacional para el filósofo, en vez de que este siga siendo el "fracasado hidalgo harapiento" de que alguna vez hablase Rorty (1996, p. 127). Así, la filosofía se convierte en una profesión y el filósofo se adjudica el status de un 'profesional' — vale decir, el técnico en los asuntos y métodos "filosóficos"- del cual, una de las primeras cosas que se espera que "haga", en el conjunto sociocultural, es "trabajar" en "lo suyo", ligando a la filosofía con la enseñanza, contribuyendo a la educación del pueblo por medio de la comunicación filosófica oficial ${ }^{8}$. Como claramente señala Logan Wilson al respecto, "the normal expectancy within the profession is that an individual entering as a recruit shall eventually become a profesor or profesor administrant" (1976, p. 97). Si esto es así, entonces el filósofo, al ser reclutado para la enseñanza, cumplirá, en la sociedad y en la cultura, una evidente función educacional.

\section{Cimentación educacional de la normalidad filosófica}

Como una herencia medieval de su historia, la filosofía, considerada antaño ancilla theologiae, era aprendida, durante buen tiempo, en función al interés teológico. Sin embargo, con el posterior proceso de secularización de la vida y la cultura en Occidente, la filosofía, al producirse una escisión entre razón y fe, se autonomiza, y se la empieza a cultivar por ella misma, sin que necesariamente sea puesta en función al interés teológico. 
Lo anterior, en Occidente abrirá las puertas, a la postre, a la profesionalización de la filosofía, y en Latinoamérica, por influjo posterior, a la futura normalidad filosófica. En adelante, el interés consistirá en "ser filósofo" como tal, erigiéndose en un esfuerzo supremo de ilustración y cultivo autónomo y desacralizado de la razón. Y lo que es aún más importante: dada la antigua vinculación tradicional de la filosofía con la educación, tal profesionalización de la filosofía, traducida en la obtención de las necesarias habilitaciones académico-profesionales para el ejercicio de la profesión de "filósofo", anduvo estrechamente ligada a la educación nacional en tanto y en cuanto "educación filosófica".

Así las cosas, obtener un título profesional en filosofía constituía, de facto, una habilitación laboral para 'enseñar' esa materia en colegios, liceos, universidades y en cuanta institución educacional exista con una naturaleza, propósitos y currículo no necesariamente emparentados o sometidos a la influencia o a los requerimientos eclesiásticos. Esto fue abriendo paso pues a una adscripción más seglar de la filosofía a los grandes objetivos educacionales nacionales ${ }^{10}$.

¿Cuál es el vínculo entre la filosofía y la educación del pueblo? Desde los griegos, la filosofía ha contribuido a la $\pi \alpha 1 \delta \varepsilon i ́ \alpha$ en tanto que encaminamiento de los humanos por la senda de determinado ideal educacional y cultural del conjunto social. La filosofía contribuye fomentando las dimensiones teóricas que le son propias: criticidad, reflexividad, radicalidad, problematicidad, interrogación, cuestionamiento. Contribuye, de este modo, a que los ciudadanos, en su formación, desarrollen estas cualidades y sean personas libres, autónomas, reflexivas y críticas $^{11}$, pero con capacidad de comprometerse - en el terreno de lo práctico- con el presente y el destino de la $\pi$ - $\lambda ı \varsigma$ y de asumir, en el terreno de la vida activa, el mismo compromiso ético que mantiene con la verdad en el campo de la vida contemplativa ${ }^{12}$.

El individuo que recibe "educación filosófica" ${ }^{13}$ puede ser libre pero capaz de ser lo suficientemente reflexivo como para asumir de modo consciente y resiliente con las crisis (Cf. Lara y Lara, 2016), su ciudadanía y su sentido de la participación. De este modo, la filosofía - lejos de constreñirse a ser un mero sistema teórico-conceptual que, por la dimensión "administrativa" de la normalidad filosófica ${ }^{14}$, queda anclado en los lindes de la Academia, enrumbado a ser un saber de solo la cultura universitaria, $y$, a la postre, privatizado como un simple "saber profesional"- democratiza su destino y puede aterrizar al conjunto sociocultural como una función ordinaria de cimentación educacional, capaz de apostar por la orientación y la formación del hombre, y capaz de desplegar, por consiguiente, una intención educacional.

En razón de lo anterior, aquella educación filosófica tendrá pues un cariz político en tanto y en cuanto suponga el fomento de algún grado de identifi- 
cación entre el individuo y los objetivos educacionales y socioculturales de la $\pi$ ó̀is.

Siguiendo este esquema, la normalización de la filosofía, según la conceptualización romeriana de 1940, supuso su profesionalización y, en consecuencia, su enseñanza más estable, orgánica, corporativa o institucional, sistemática y de un jaez laical, incorporada a un sistema educacional orgánico nacional, en los centros de educación superior y secundaria. A mi juicio, este desarrollo fue enteramente beneficioso tanto para la propia filosofía cuanto para el sistema educacional al cual ella se adscribió o se incorporó, propiciando un giro institucional de la filosofía en Latinoamérica. En efecto, normalizarse le significó a la filosofía la posibilidad de, vía la educación filosófica, convertirse en tributaria de la educación del pueblo. Se podía incorporar a un proyecto educacional, a un propósito social formativo. Y, con ello, la filosofía misma se adjudica de un carácter educativo en el conjunto social. En la esfera pública, ese será, por consiguiente, su lugar normal, su ámbito natural, digamos asi ${ }^{15}$.

Si esto es así, entonces estamos ante una normalidad filosófica cimentada educacionalmente y en movimiento, lo cual le da un carácter abierto. Se trata de un proceso en las ideas que, por mucha autonomía que se pretenda que tenga, de algún modo tiene algún referente exterior a la filosofía, en los procesos histórico-sociales. Si la normalidad filosófica tiene un carácter abierto, llega hasta nuestros días teniendo a los cuestionamientos que se formulen a la filosofía como principal referente y signo de contradicción, lo cual vehiculizará y posibilitará que tal normalidad, fenómeno de indiscutible y evidente raigambre educacional, sea un reto permanente, tanto en el plano de su interpretación cuanto en el plano de su concreción más que un mero logro ya plenamente consumado.

\section{La doble dimensión educacional de la normalidad filosófica}

Llegados a este punto de nuestro discurso, es conveniente hacer aquí un distingo entre dos planos de la normalidad filosófica, que aquí denominamos, respectivamente, conceptual o formativo y administrativo. Ambos planos oscilan entre la normalización de un discurso filosófico y la normalización de la filosofía como una actividad. Veamos.

Con el primer plano, el formativo o conceptual, estamos entendiendo a la filosofía como un proceso de producción de bienes filosóficos: filosofemas, teorías y planteamientos. Y, si se usa la expresión "normalidad filosófica" con esta acepción, entonces se está haciendo alusión a que un discurso, una determinada concepción filosófica o manera de entender la filosofía se difunde en nuestra cultura, incluyendo en dicha concepción los planteamientos varios acerca de los múltiples problemas filosóficos. Implica, pues, los procesos de producción de bienes filosóficos. 
Este plano conceptual, a su vez, supone un doble aspecto: por un lado, la normalización de un discurso-repetición, expropiado; ajeno, en este caso el europeo, porque no había todavía un nivel de discurso propio en Latinoamérica. Y, por otro lado, supone un nivel de normalización posterior cuando los pensadores latinoamericanos empiezan a asumir una actitud más refractaria y crítica frente a ese discurso inicial; lo cuestionan, lo tildan de colonialista y buscan romper con él y sus raíces. En este caso, estamos hablando de que se habría de producir la normalización de un discurso propio, que busca autenticidad; lo cual pasa, pues, por postular una ruptura con el discurso oficial.

El segundo plano, el administrativo, es el evento inicial de la normalidad filosófica. Tomada en este sentido, la objetivación "normalidad filosófica" se refiere a la conversión de la filosofía en una profesión, en un proceso análogo al que acaeció en el Viejo Mundo. Vale decir, se refiere al ejercicio con carácter de profesión remunerada de esta actividad. En virtud de esta instalación académico-profesional de la filosofía, esta podía enseñarse de manera institucional laical, sirviendo, además, como plataforma giratoria o espacio para el filosofar y la administración de un filosofar en el sentido conceptual que más atrás establecíamos. Este segundo sentido implica los procesos de circulación y consumo de los bienes filosóficos.

El primer sentido, el conceptual o formativo, tiene un carácter más teórico, puesto que se refiere a la filosofía como actividad intelectual productora de un saber de determinadas características. Pero también se refiere a las concepciones que subyacen a dicha actividad. Entretanto, el segundo sentido posee un carácter más administrativo, por así decirlo, puesto que implica un ejercicio institucional de la filosofía, vinculado a entidades educacionales o que están involucradas en el sistema formal oficial de educación del pueblo; las cuales cumplen, pues, una clara función educacional.

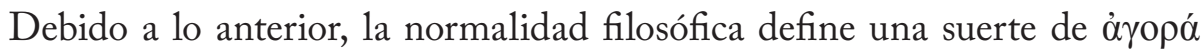
o de plataforma giratoria en la cual, a pesar de que la ley sea la conexión y el intercambio, todos los pensadores que tienen acceso a los procesos de producción, circulación y consumo de los productos filosóficos gocen de la más amplia libertad intelectual para filosofar. Este es un acto libre y liberador a un tiempo, una suerte de teoría de la liberación humana ${ }^{16}$, que nos interpela y nos obliga a

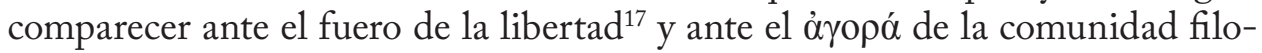
sófica dialógica.

Ambas dimensiones de la normalidad filosófica, como fácilmente se podrá apreciar, poseen un carácter educacional, puesto que implican la adscripción de la filosofía a un sistema educacional; incorporándose a un esquema educacional nacional, involucrándose con los propósitos educacionales; contribuyendo, desde su propia perspectiva, a tales fines. Si la filosofía es busca incondicional de la verdad, entonces esta, como señala Jaspers, debe también poder ser transmitida 
(Cf. Jaspers 1959, p. 394). Asimismo, se introduce, en el imaginario sociocultural, algún concepto de filosofía, una manera de entenderla, adjudicándose, al mismo tiempo, de un determinado rol técnico-profesional que genera empoderamiento, trabajo, preparación y compromiso con la educación del pueblo y, en general, con la formación del espíritu nacional.

\section{Nota ontológica}

La đódıৎ misma está en movimiento ${ }^{18}$, y ese movimiento repercute también en la objetivación "normalidad filosófica". Esta, tal vez posea un substrato permanente - el cual vendría a estar dado por la persistente e irrenunciable vocación de la filosofía por la busca de la verdad, teniendo como enseña la impregnación de todas las dimensiones de la vida sociocultural. No obstante, sus accidentes serán movibles y constituirán la dimensión más próxima, externa o perceptible para nosotros. Ambas dimensiones son, pese a movibles, objetivables en el orden del discurso.

Las categorías del pensamiento y del lenguaje están en movimiento porque la realidad referente lo está (Cf. Berger y Luckmann 1998, p. 52ss). Por consiguiente, una objetivación como "normalidad filosófica" no puede estar exceptuada de esa determinación. Tiene carácter movible y abierto porque los indicadores de normalidad objetivados por Romero en 1940 están en movimiento, no son estáticos, están sujetos a determinaciones temporales. Cada indicador se actualiza permanentemente, de acuerdo con los desafíos y recursos propios de cada tiempo y cultura.

Análogamente, una categoría como "normalidad filosófica" es una objetivación que, si bien fue propuesta por Francisco Romero en el primer tercio del siglo XX, describe un estado por el que atravesaba la filosofía en Latinoamérica en aquel tiempo. Ya hemos explicado lo que ella significaba: la conversión de la filosofía en una común función cultural. Esto implicó que la filosofía pasó a formar parte de la esfera pública de entonces, ganando espacios socioculturales para sí que posibilitarían un grado de apertura al conjunto sociocultural, con prescindencia de la mera esfera de influencia e injerencia eclesiástica. Y esta objetivación se ve enriquecida por el aporte de las generaciones subsiguientes de pensadores, quienes tienen que asumir y enfrentar, de modo permanente, todos los retos que supone la asunción de la filosofía como una 'función ordinaria de la cultura'. Ni el mismo concepto de filosofía, inicialmente propuesto por los pensadores griegos — por lo demás, desde un inicio disímiles también entre sí- ha permanecido inmóvil y sin diversificarse y enriquecerse con las distintas experiencias de las generaciones posteriores de pensadores en el curso de la historia del pensamiento ${ }^{19}$. 
Precisamente, en este sentido, la inserción de la filosofía en el complejo cultural como una "función ordinaria" hará que cada cierto tiempo los indicadores del estado de normalidad filosófica se interpelen, enriquezcan o problematicen. En una palabra, que se actualicen. El mismo sistema educacional, o los objetivos educacionales nacionales, están sujetos a revisión y actualización. No son estáticos. En todos los casos, no debemos perder de vista la naturaleza de la filosofía, un saber crítico y problematizador sin condiciones ${ }^{20}$ por excelencia.

Por ejemplo, la voluntad de aproximación dialógica y de establecer redes intelectuales entre los filósofos fue uno de los indicadores señalados por Romero en su diagnóstico de 1940. La exigencia de busca dialéctica de la verdad generará la necesidad de un mayor acercamiento entre los interesados, avivándose la conexión de que hablaba Romero en 1959, mediante la creación de espacios o escenarios en cuyo seno pueda concretarse tal conexión. Cada tiempo los reclamará, pero se acomodarán/enfrentarán/las verán con las circunstancias o condicionantes socioculturales, económico-políticos e ideológicos reinantes. Y esto incluye al aparato educacional nacional.

Todo lo anterior hará que la normalidad filosófica sea siempre una pregunta abierta. La filosofía no tiene la existencia comprada en el aparato cultural, y más bien, cada cierto tiempo debe justificar y defender los fueros que en su historia ha ganado - y que le han generado derecho propio-, porque siempre se la cuestiona; especialmente con cada nuevo avance, logro o hallazgo de la investigación científica.

La pregunta por la normalidad filosófica, en consecuencia, nos envuelve, nos implica, porque es el evento preguntado; constituye una realidad de la que, en algún sentido o de alguna manera, formamos parte, y no somos meros espectadores más o menos neutrales. Ella es parte de nosotros en el conjunto social. Ella y nosotros conformamos un todo indisoluble, porque dicha normalidad filosófica, como evento humano que es, es parte de la realidad social ${ }^{21}$. Es un evento próximo a nosotros, del cual, se diga lo que se diga, muestra con toda evidencia la realidad de algo que, no obstante, se nos ha metamorfoseado en un problema. Nos pone en evidencia una presencia. Es la presencia de la filosofía. De algún modo o en algún sentido, esta es una presencia, y forma parte de nuestra cotidianeidad. Carecería de sentido preguntarnos por la normalidad filosófica si la misma filosofía, de algún modo o en algún sentido, fuese, entre nosotros, algo sin significación. ¿Cuál es su mismidad? Precisamente, aquella pregunta es en rigor, una pregunta por la misma filosofía, que-está-aquí/ahí.

\section{Conclusiones}

El examen precedente ha mostrado que la normalidad filosófica implicó la conversión de la filosofía en una "función ordinaria de la cultura". Esto planteó el 
problema de cuál era ese espacio "propio" que la filosofía podía desempeñar en el conjunto sociocultural y si suponía la asunción de algún "rol social”. Se establece que ese rol, en la cultura, tiene raigambre educacional, lo cual no anula sino más bien, encauza la naturaleza de la filosofía de ser busca desinteresada pero comunicable del conocimiento. Así, se establece que la filosofía comenzó a ser enseñada de manera regular, sistemática, profesional y, sobre todo, con un espíritu más laical y menos dependiente de control y/o influencia ideológica eclesiástica directa, porque esto último favorecía el libre examen y el librepensamiento.

Aparecía la exigencia de que se la enseñase con este espíritu en las instituciones universitarias, desde donde irradiará su esfera de influencia pedagógica hacia las instituciones educativas secundarias de la educación básica; incorporándose a los sistemas educacionales oficiales, quedando involucrada en las intenciones nacionales para brindar educación en favor de los pueblos latinoamericanos. Con ello, la normalidad filosófica adquiría un carácter educacional en una doble dimensión: administrativa y formativa, lo cual le adjudicaría el atributo de ser también un evento educacional, involucrando una enseñanza filosófica que, si bien constaba de contenidos aprendidos de Europa, estuvo puesta más en función de consolidar los indicadores objetivados por la categoría "normalidad filosófica" y de hacer ingresar a Latinoamérica al cauce filosófico mundial.

La evidencia muestra que la normalidad filosófica se configura como un fenómeno educacional abierto y movible, porque los indicadores del estado de normalización enumerados por Romero en 1940 tienen presencia permanente pero actualizable a la vez en el contexto filosófico latinoamericano y definen la calidad de evento educacional de tal normalidad. En consecuencia, en virtud a este carácter abierto, la presencia o incidencia actual (siglo XXI) de tales indicadores no tiene necesariamente por qué ser la misma que aquella del primer tercio del siglo XX.

\section{Notas}

$1 \mathrm{El}$ presente artículo constituye un resumen apretado de una parte del Capítulo 1 de la tesis titulada La normalidad filosófica en Francisco Romero como normalidad educacional de la fllosofía en Latinoamérica que será presentada por el autor para optar el grado académico de Magister en Filosofía, mención Historia de la Filosofía, en la Unidad de Posgrado de la Facultad de Letras y Ciencias Humanas de la Universidad Nacional Mayor de San Marcos.

2 De hecho, hemos examinado antes (Cf. Sánchez, 2015) que Romero mismo fue un normalizador de la filosofía en Latinoamérica, y su dedicación a tiempo completo a la filosofía constituyó un testimonio actante en primera persona del proceso de la normalidad filosófica latinoamericana. 
3 Hay que advertir que no fue aquella, empero, la primera ocasión en que Romero se habría de referir al tema de la normalidad filosófica: ya lo había hecho en 1934, en un homenaje a Manuel García Morente, en la sede del PEN Club de Buenos Aires (Cf. Romero, 1950).

4 Esto es, como decía Zea, “que trascendía los cenáculos académicos” (Cf. Zea, 1983, p. 171). O incluso, como señala Augusto Salazar Bondy, implica la aceptación de que la filosofía es "un ejercicio intelectual al alcance de mentalidades más o menos comunes" (Cf. Salazar, 1968, p. 58).

5 Es decir, aquello que Romero denominó en otro lugar la conexión americana (Cf. Romero, 1959).

6 Ver $§ 5$, en este escrito.

7 Aislándose y/o desentendiéndose del resto del conjunto sociocultural, en una suerte de celibato social, como lo ha denominado Oncina (2009, p. 21); el cual, en nuestra opinión, sería un signo de burocratización sociocultural del filósofo.

8 Este hecho, al decir jocoso de Vattimo, resulta ventajoso para el filósofo porque, si no, como señala, "no sé quién nos pagaría" (Cf. Vattimo, 2012, p. 56).

9 Aun cuando, como señala Ranovsky (2011, p. 55ss), ser profesional y ser profesor sean actividades opuestas, pensamos que en el "profesor de filosofía" se sintetizan ambas dimensiones, como ocurrió en el caso del propio Francisco Romero.

10 Así, por ejemplo, es fácil ver cómo en los escritos hegelianos sobre educación (Cf. Hegel, 1991) la filosofía cumple un papel de primer orden en la educación del pueblo.

11 Que pasen a formar parte del "Tribunal Supremo" de que, irónicamente, habla Gustavo Bueno (1999, p. 92), sin espíritu de gremio o de gueto, de espaldas a la realidad social.

$12 \mathrm{Al}$ respecto, es de mucha utilidad la distinción arendtiana entre vida activa y vida contemplativa (Cf. Arendt, 1996 y 2002).

13 Para Marrou (2000, p. 102), el fundador de esta fue Platón.

14 Cf. $\$ 5$ de este escrito.

15 No se nos escapa, empero, las dificultades que esta institucionalización plantea, como, por ejemplo, qué ha de ser la filosofía, cuál sería epistémicamente su identidad y su lugar en el contexto de la cultura. Este asunto queda planteado, por ejemplo, en el kantiano conflicto de las facultades (Cf. Kant, 1992 [1798], y Derrida, 1984). Tema este que, por su complejidad, no podremos abordar aquí.

16 Cf. Dunham, 1967.

17 La libertad intelectual del filósofo fue el tema de una importante Mesa Redonda auspiciada por la Unesco, en 1952, con ocasión de celebrarse, en México, el III Congreso Interamericano de Filosofía (Cf. Unesco, 1952).

18 Que, a su vez, problematiza y mueve a la misma $\pi \alpha \imath \delta \varepsilon i ́ \alpha$ (Cf. Romero y Wichazki, 1989).

19 No puede ser para menos que con una categoría como "normalidad filosófica" acontezca lo dicho, porque de por medio está implicado el carácter social del lenguaje. Al decir de Schaff (1967, p. 250) "el lenguaje es aquello en lo cual se hallan encerradas y establecidas las experiencias y el saber de las generaciones pasadas” (y las por venir, añadiríamos nosotros).

20 Un saber sin restricciones ni parámetros, que encarna la más alta libertad de pensamiento y, como señala Derrida (2010, p.10ss), de cuestionamiento, así como del derecho a decir públicamente un pensamiento y un saber de la verdad, en el contexto de una universidad que, en tanto creadora de conocimiento ("La universidad tiene la misión de buscar la verdad en 
la comunidad de investigadores y discípulos”, señala Jaspers [Op.Cit. p. 392] por su parte), tendría también una misión unificadora del saber, en medio de la diversificación producto de la especialización del conocimiento científico (Cf. Heidegger 1967, p. 77).

21 En este punto, debemos recordar, con el sociólogo argentino Sergio Bagú (1981, p. 11ss), que la realidad social y el conocimiento de dicha realidad social conforman una dicotomía indisoluble.

\section{Referencias Bibliográficas}

Arendt, H. (1996). La condición humana, Barcelona, Editorial Paidós.

-. (2002). La vida del espiritu, Barcelona, Editorial Paidós.

Badiou, A., Žižek S. (2011). Filosofía y actualidad. El debate. Buenos Aires, Amorrortu Editores.

Bagú, S. (1981). Tiempo, realidad social y conocimiento. Propuesta de interpretación. México, Siglo Veintiuno Editores.

Becher, T. (2001). Tribus y territorios académicos. La indagación intelectual y las culturas de las disciplinas, Barcelona, Gedisa Editorial.

Berger, P. L., Luckmann T. (1998). La construcción social de la realidad, BuenosAires, Amorrortu Editores.

Bueno Martínez, G. (1999). ¿Qué es la filosofía? El lugar de la filosofía en la educación. El papel de la flosofía en el conjunto del saber constituido por el saber político, el saber cientifico y el saber religioso de nuestra época. Oviedo, Pentalfa Ediciones.

Canfora, L. (2002). Una profesión peligrosa. La vida cotidiana de los filósofos griegos. Barcelona, Editorial Anagrama.

Collins, R. (2005). Sociología de las filosofías. Una teoria global del cambio intelectual, Barcelona, Editorial Hacer.

Derrida, J. (1984). La filosofía como institución. Barcelona, Ediciones Juan Granica.

- (2010). La Universidad sin condición. Madrid, Editorial Trotta.

Dunham, B. (1967). La filosofía como liberación humana. Barcelona, Ediciones Península.

Hegel, G.W.F. (1991). Escritos Pedagógicos. Madrid, Fondo de Cultura Económica.

Heidegger, M. (1967). ¿Qué es Metafísica? Buenos Aires, Ediciones Siglo Veinte.

Jaspers, K. (1959). “La idea de la universidad”. En: Varios, La idea de la universidad en Alemania buenos Aires, Editorial Sudamericana.

Kant, I. (1992) (1798). La contienda entre las facultades de Filosofía y Teología. Madrid, Consejo Superior de Investigaciones Científicas.

Lara Nieto, M. C., Lara Lara, F. (2016). “Qué es, qué podemos hacer y qué nos cabe esperar con la enseñanza de la filosofía en el siglo XXI”. En: Rafael Orden, Juan García Norro y Emma Ingala, Diotima o de la dificultad de enseñar filoso- 
fía. Madrid, Escolar y Mayo Editores/Facultad de Filosofía de la Universidad Complutense.

Marrou, H. (2000). Historia de la educación en la Antigüedad. México, Fondo de Cultura Económica.

Oncina Coves, F. (2009), "La filosofía clásica alemana y su idea de la Universidad: ¿un anacronismo viviente?”. En: Oncina Coves, Faustino (Ed.), Filosofía para la universidad, flosofía contra la universidad (de Kant a Nietzsche). Madrid, Editorial Dykinson/Universidad Carlos III de Madrid.

Ranovsky, A. (2011). Filosofía del docente filósofo. Buenos Aires, Colisión Libros.

Romero Delgado, F. (1950). "Sobre la normalidad filosófica. Palabras a M. García Morente”. En su: El hombre y la cultura. Buenos Aires, Espasa-Calpe Argentina.

-. (1957). "La filosofía, la cultura y el hombre”. En su: Filósofos y Problemas, Buenos Aires, Ed. Losada.

—. (1959). "Discurso de clausura del Sexto Congreso Interamericano de Filosofía". En: Revista Brasileira de Filosofa. Vol. IX, Fasc. IV, Oct.-Nov.-Dic.

- (1961). "Sobre la filosofía en Iberoamérica". En su: Filosofía de la persona y otros ensayos de filosofía. Buenos Aires, Ed, Losada.

Romero, C., Wichazki, M. (1989). "Polis y paideia”. En: Ponencias (2a Parte) del V Congreso Internacional de Filosofía Latinoamericana. Julio de 1988. Bogotá, Centro de Enseñanza Desescolarizada de la Facultad de Filosofía de la Universidad Santo Tomás.

Rorty, R. (1996), "La profesionalización de la filosofía y la cultura trascendentalista”. En su: Consecuencias del pragmatismo, Madrid, Editorial Tecnos

Salazar Bondy, A. (1968). ¿Existe una filosofía de nuestra América? México, Siglo XXI Editores, S.A.

Sánchez Paredes, C. (2015). Francisco Romero como normalizador de la filosofía en Latinoamérica. Tesis de Licenciatura en Filosofía, UNMSM.

Schaff, A. (1967). Lenguaje y conocimiento. México, Editorial Grijalbo.

Unesco (1952), El peligro de la libertad intelectual. Tercer Congreso Interamericano de Filosofía. Mesa Redonda. México, Universidad Nacional Autónoma, Facultad de Filosofía y Letras.

Vattimo, G. (2012). Vocación y responsabilidad del filósofo. Barcelona, Editorial Herder.

Wilson, L. (1976). The academic man. A study in the sociology of a profesion. New York, Octagon Books.

Zea, L. (1983). Romero y la normalidad filosófica latinoamericana. En: Arturo Ardao et al., Francisco Romero, maestro de la filosofía latinoamericana. Caracas, Secretaría de la Sociedad Interamericana de Filosofía. 